\title{
Der kulturelle Kontext von Schlichtung Zum Stand der Schlichtungs-Forschung in der Rechts-Anthropologie
}

\author{
Werner Notbdurft und Thomas Spranz-Fogasy
}

Zusammenfassung: Die rechtsanthropologische Literatur zum Thema Schlichtung wird auf ihre Fruchtbarkeit für eine gesprächsanalytische Untersuchung von Schlichtung ausgewertet. Rechtsanthropologische Arbeiten liefern insbesondere Ergebnisse zu folgenden vier Aspekten von Schlichtung: Schlichtung als eines von unterschiedlichen Verfahren der Konfliktbearbeitung (das kulturelle Paradigma), Schlichtung als Phase in der komplexen Bearbeitungsgeschichte eines Konflikts (das Syntagma der Bearbeitungsgeschichte), Schlichtung als interaktiv charakteristisches Muster der Konfliktbearbeitung (Prozeß-Modelle) und die Interdependenz von Schlichtung als effektivem Bearbeitungsverfahren mit kulturellen Randbedingungen (der Bedingungszusammenhang von Schlichtung). Abschließend werden Implikationen der rechtsanthropologischen Befunde für eine gesprächsanalytische Untersuchung von Schlichtung angedeutet.

Summary: The cultural context of mediation. On the state of mediation research in legal anthropology.

The literature on legal anthropology dealing with the subject of mediation is examined as to its relevance to an investigation on mediation in terms of conversational analysis. The literature contains findings on the following four aspects of mediation: mediation as one of various techniques of conflict resolution; mediation as a phase in the complex history of a conflict; mediation as a characteristic interactive pattern of conflict resolution; and the dependence of mediation as an effective resolution technique on certain cultural conditions. In conclusion the implication of these findings from legal anthropology for an investigation on mediation in terms of conversational analysis are touched on.

\section{Vorbemerkung}

Die vorliegende Arbeit entstand im Rahmen eines Forschungsprojekts ,Schlichtung Gesprächs- und Interaktionsanalyse eines Verfahrens zur Lösung sozialer Konflikte". 1 Ziel des Projekts ist es, die interaktive Dimension von Schlichtung zu erfassen, das allgemeine Handlungsmuster, das Schlichtungsgesprächen zugrundeliegt, festzustellen und die charakteristischen Kommunikationsprobleme, die beim Schlichten bewältigt werden müssen, aufzudecken. Insbesondere in der Rechtsanthropologie findet sich eine Vielzahl von Arbeiten, die das Schlichten von Streit zu ihrem Untersuchungsgegenstand machen. Die Fruchtbarkeit solcher rechtsanthropologischen Studien für eine gesprächsanalytische Untersuchung von Schlichtung soll in der vorliegenden Arbeit geprüft werden.

1 Das Projekt wird am Institut für deutsche Sprache (IdS) (Mannheim) durchgeführt und ist Bestandteil eines umfangreichen Forschungsprogramms zur typologischen Bestimmung komplexer gesellschaftlicher Handlungsmuster. 
Der vorgenommenen Sichtung lagen ca. 40 rechtsanthropologische Arbeiten zum StreitSchlichten zugrunde, von denen 27 in die Darstellung eingingen. Ausgangspunkt der Sichtung waren die Übersichtsartikel von Collier ${ }^{2}$ und Spittler ${ }^{3}$.

\section{Die Einbindung der Scblicbtungs-Thematik in den recbtsantbropologiscben Diskussionszusammenhang}

Noch 1977 stellt Gulliver fest, daß Schlichtung im Bereich rechtsanthropologischer Forschung als Untersuchungsobjekt ein Schattendasein fristet:

mediation still remains an inadequately understood process and by no means only in the single field of industrial relations. Especially in anthropology the subject has been grossly neglected, along with the whole process of negotiations. "4

Nur randständig würde Schlichtung in Untersuchungen behandelt und dann unter , useless aphorisms" als ,,informal procedures", ,private conciliation" oder "the judicial process in one of its prenascent forms". In dem grundlegenden Werk von Pospisil "Anthropology of Law" ${ }^{65}$ findet sich für Schlichten kein Index-Eintrag ${ }^{6}$.

Es bedarf wohl der Vergegenwärtigung der Forschungsgeschichte der Rechtsanthropologie und ihrer Kontroversen, um diese Marginalität von Schlichtung verstehen zu können:

Die Rechtsanthropologie ist lt. Comaroff $\&$ Roberts $^{7}$ geprägt von dem Streit zwischen einem normativ orientierten, legalistischen Ansatz und einem interpretativen, konflikttheoretischen Ansat $z^{8}$.

Der normativ orientierte Ansatz hat seinen Ausgangspunkt in der Arbeit von Maine „Ancient Law"9 ; Maine entwickelt in einem evolutionistischen Ansatz den aktuellen Rechtszustand aus primitiveren Formen heraus, die sich in ihrer Primitivität in anderen Kulturen noch auffinden lassen, gleichsam als Fossilien. "Recht", insbesondere in der anglo-amerikanischen Version, wird als natürliche analytische Kategorie betrachtet, auf

2 Jane F. Collier, Legal Processes, in: Annual Review of Anthropology 4 (1975), 121-144.

3 Gerd Spittler, Streitregelung im Schatten des Leviathan. Eine Darstellung und Kritik rechtsethnologischer Untersuchungen, in: ZfRSoz 1 (1980), 4-32.

4 P. H. Gulliver, On Mediators, in: Ian Hamnett (ed.), Social Anthropology and Law, London $1977,15-52$ (44).

5 Leo Pospisil, Anthropology of Law, New York 1971.

6 Man könnte einwenden, daß Gullivers Bewertung eine Verwechslung von Begriff und Phänomen zugrundeliegt. In der Tat wird häufig das Phänomen ,Schlichtung unter dem Begriff ,Aushandlung' (,negotiation') abgehandelt. Da Gulliver dies erklärtermaßen tut, kann ihm selbst eine solche Verwechslung allerdings nicht unterstellt werden. Seine Beurteilung ist ganz offenbar auf das Phänomen ,Schlichtung' bezogen, unabhängig davon, mit welchem analytischen Prädikat es behandelt wird.

7 John Comaroff/Simon Roberts, Rules and Processes, Chicago, London 1981

8 Zur Darstellung dieses Konfliktes vgl. Laura Nader (ed.), The Ethnography of Law. American Anthropologist (Special Publication) vol. 67, 3-32.

9 H. Maine, Ancient Law, London 1861. 
deren Grundlage empirisch beobachtbare Strukturen und Prozesse analysiert werden können. Die Definition von Recht erfolgt über Sanktion, Autorität und Verpflichtung. Entsprechend der aktuellen Rechtsqualität als Regelsystem mit Sanktionscharakter wird rechtliches Verhalten als normativ vorgeschriebenes und administrativ kontrolliertes betrachtet.

Der Konzentration dieses Ansatzes auf das positive Recht ist geschuldet, daß Schlichtung als ein Verfahren, das sich gerade nicht zentral auf dieses Recht stützt, außerhalb des wissenschaftlichen Interesses blieb.

Die Kritik an diesem Ansatz seitens der Konflikttheorie setzte vor allem an folgenden Punkten an ${ }^{10}$ :

1. In anderen Kulturen gibt es keine Analoga zu anglo-amerikanischen Rechtskategorien.

2. Im normativen Ansatz werden die Kategorien theoretisch als wirksam gesetzt, ohne die Varianz und die Bedingungen ihrer gesellschaftlichen Wirksamkeit empirisch zu untersuchen. Insbesondere ist nach Starr \& Ynguesson ${ }^{11}$ das Konzept sozialer Struktur und sozialer Ordnung auf die Metapher des Gleichgewichtszustandes verkürzt worden und damit das Spektrum sozialer Ordnungsherstellung eingeschränkt und seiner Dynamik beraubt worden.

3. Die analytischen Prädikate sind ,naive" Prädikate, d. h. nicht aus einer Theorie über die Rechtswirklichkeit abgeleitet, sondern aus dem Sprachgebrauch der Rechtspraktiker übernommen. Insbesondere erzeugt die Verwendung der anglo-amerikanischen Rechtskategorien ErgebnisVerzerrungen.

4. Ein empirisch bestimmbarer Unterschied zwischen Regeln mit genuin rechtlichem Charakter und solchen mit moralischem, ethischem, gruppenspezifischem Charakter läßt sich nicht nachweisen $^{12}$.

Vor allem wegen der Hypostasierung einer bestimmten Rechtsauffassung als wissenschaftliches Konzept erfolgt ${ }^{13}$ einerseits die Generierung irreführender Fragen und andererseits die Eliminierung wichtiger Untersuchungsgegenstände (z. B. wird meist die politische Natur der Rekrutierung von Dritten (statt ihrer juristischen Qualität) ausgeblendet).

Dem normativen Ansatz entgegen steht ein Konzept, das die sozialen Prozesse der Bearbeitung gesellschaftlicher Konflikte als seinen Untersuchungsgegenstand begreift. Ausgangspunkt dieses Ansatzes ist die Untersuchung von Malinowski „Sitte und Verbrechen bei den Naturvölkern “14. Gefragt wird danach, durch welche Institutionen, Bräuche etc. die soziale Ordnung in einer Gemeinschaft hergestellt bzw. wiederhergestellt wird. Nicht die präsupponierte soziale Norm oder Regel, die Stabilität sicherstellt,

$10 \mathrm{Vgl}$. Comaroff/Roberts (N. 7).

11 June O. Starr/Barbara Yngvesson, Scarcity and Disputing: Zeroing-in on Compromise Decisions, in: American Ethnologist 2 (1975), 553-566.

12 Das soll natürlich nicht heißen, daß sich nicht empirisch positives, gesetztes Recht nachweisen ließe. Das Argument zielt aber darauf ab, daß sich dieses positive Recht stets weiter auf andere - vornehmlich ethische - Kategorien zurückführen läßt und sich die Faktizität des Juristischen rekonstruktiv auflösen läßt. Die soziale Genese juristischer Normen und Verfahren ergibt sich dann als cigener, rechtssoziologischer Untersuchungsgegenstand.

13 So Comaroff/Roberts (N 7).

14 Bronislav Malinowski, Crime and Custom in Savage Society, London 1926 (dt. Obers.: Sitte und Verbrechen bei den Naturvölkern) 
ist Untersuchungseinheit, sondern der soziale Aushandlungsprozeß, durch den Ordnung zustandekommt und Konflikte bearbeitet werden. Dieser soziale Aushandlungsprozeß wird It. Malinowski vor allem durch Merkmale der Sozialbeziehung (Verpflichtungen, Erwartungen und Reziprozität) und durch die Handlungsperspektiven der Beteiligten (Interessen, Ziele, Relevanzen) gesteuert. Soziale Regeln tauchen nicht als Handlungsdeterminanten, sondern als Aushandlungsgegenstände auf. Bei Malinowski ist Konflikt-Bearbeitung immer schon über Sozialbeziehungen etc. strukturell - quasi vorab - geregelt; nicht in den Blick kommt, was passiert, wenn diese Mechanismen gerade nicht mehr funktionieren. Offenbar untersuchte Malinowski eine Ethnie, in der diese Mechanismen besonders gut funktionierten, so daß darüberhinausgehende Formen der Konflikt-Bearbeitung nicht (oder jedenfalls nicht auffällig) auftraten. Symbolisch ablaufende Prozesse, wie z. B. Schlichtung, kommen so nicht in den Blick.

Die Kontroverse zwischen diesen beiden Ansätzen greift vor allem an jenen Punkten an, die von den Ansätzen jeweils als ihre Besonderheit behandelt werden: Dem Aushandlungsansatz ist von Anhängern des normativen Ansatzes vorgeworfen worden, den Gegenstand der Rechtsanthropologie aufzulösen und dessen Spezifik, ,,das Rechtliche“, nicht angemessen zu erfassen. Während dieser Vorwurf der konzeptuellen Breite umstandslos zurückgegeben werden kann mit dem Vorwurf allzu enger Konzeptionsbestimmung, wiegt eine andere Kritik schwerwiegender: Im Aushandlungsansatz werde ein simplizistisches Modell des Handelnden entworfen, der allzusehr als Gewinn-Maximierer und strategisch Handelnder vorgestellt werde. Nach Comaroff \& Roberts ${ }^{15}$ besteht in der Tat die Gefahr, daß in diesem Ansatz die normative Orientierung des Handelnden übersehen wird.

Comaroff \& Roberts schlagen als Mittelweg eines Untersuchungsprogramms vor, die kulturell unterschiedlichen Mittel der Konfliktaustragung in ihrer sozialen Regelhaftigkeit und vor ihrem Werthintergrund zu analysieren.

Man bemerkt, daß Schlichtung auch in diesem Vorschlag nicht als eigenständiger Untersuchungsbereich vorkommt, sondern in seiner Einbettung in die komplexeren Begriffsfelder ,Konfliktbearbeitung', soziale Regelhaftigkeit' und ,kulturelle Werte‘. Bei der Literatursichtung wird sich zeigen, daß diese Einbettung, die aus der geschilderten Kontroverse resultiert, charakteristisch für die Aussagen und Ergebnisse über Schlichtung ist.

\section{Allgemeine Charakterisierung der Forschungslage für Scblichtung}

Was unter diesen wissenschaftsgeschichtlichen Umständen für Interessen eines gesprächsanalytischen Forschungsvorhabens über Schlichtungs-Gespräche von einer Sichtung rechtsanthropologischer Literatur zum Schlichten erwartet werden kann, ist zum einen der Aufweis kultureller Variabilität von Schlichtungs-Formen und zum anderen die Darstellung der Einbettung von Schlichtung in den übergreifenden sozialkulturellen Lebenszusammenhang. 
Die Sichtung einschlägiger Literatur wird durch den Umstand erleichtert, daß sich die meisten Autoren eines ähnlichen Schlichtungs-Begriffs bzw. einer ähnlichen Arbeitsdefinition bedienen. Abgehoben wird durchweg auf die triadische Rollen-Struktur, den vorgängigen Konflikt-Charakter und das Handlungsziel der Einigung. Beispiele:

,Mediation refers to the range of methods by which third persons seek to resolve a dispute without imposing a binding decision." 16

"In negotiations there may be a third party who, though he has no ability to give a jugdement, acts in some way as a facilitator in the process of trying to reach agreement."17

,Mediation $\ldots$ involves a third party who intervenes in a dispute to aid the principals in reaching an agreement. “ 18

Es scheint in der Tat sinnvoll, auf weitergehende Festlegungen in einer Schlichtungsdefinition zu verzichten, um die Gefahr eines kulturellen Vorurteils möglichst gering zu halten. So vermutet Gulliver ${ }^{19}$ bereits für das so evidente Schlichtungs-Merkmal der Neutralität der Schlichter-Rolle, daß es sich hierbei eher um ein Element des kulturellen Überbaus von Schlichtung (der Ideologie von Schlichtung) handelt als um ein allgerneines, kulturübergreifendes Definitions-Merkmal. Ähnlich problematisiert Cohen (1967) die für Schlichtung angeblich charakteristische Freiwilligkeit der Unterwerfung unter eine Einigung: Die Bestimmung von Schlichtung als ,... the process by which a third person intervenes between two contending parties in an effort to reconcile them

- fails to recognize the extent to which American mediators, at least in disputes affecting the public interest, actually bring pressure on reluctant parties to accept an unsatisfactory settlement." 20

Entsprechenden Bestimmungen von Schlichtung, wie sie sich z. B. bei Spittler ${ }^{21}$ oder Bohannan ${ }^{22}$ finden, ist daher bereits mit Skepsis zu begegnen.

Übereinstimmungen finden sich in den ausgewerteten Arbeiten hinsichtlich des methodischen Vorgehens: Sie sind weitgehend als Feldstudien mit nicht-standardisierten Frhebungsverfahren (teilnehmende Beobachtung, unstrukturierte Interviews) angelegt. Die Beobachtungsdaten sind in unterschiedlicher Weise präsent, z. T. als informell geführte Protokolle, z. T. als Tonbandaufnahmen, z. T. als Filmmaterial. Die Auswertung erfolgt interpretativ. Quantitative Auswertungen fanden sich in den gesichteten empirischen Arbeiten nicht. Datenquellen sind oft aber auch die Materialien anderer Autoren; sei es um Reanalysen durchzuführen, seı es, um einigen Ansätzen ein empirisches Fundament zu geben.

Die Sichtung der Literatur erbrachte Ergebnisse, die sich in folgende vier Gruppen gliedern lassen:

16 Jerome Alan Cohen, Chinese Mediation on the Eve of Modernization, in: David Buxbaum (ed.), Traditional and Modern Legal Institutions in Asia and Africa, Leiden 1967, 54-76 (54).

17 Gulliver (N. 4), 15 .

18 Laura Nader/Harry F. Todd (eds.), The Disputing Process. Law in Ten Societies, New York $1978,10$.

19 Gulliver (N. 4), 45.

20 Cohen (N 16), 54.

21 Spittler (N 3).

22 Paul Bohannan, The Differing Realms of Law, in: Nader (ed.) (N 8), 33-42. 
- Ergebnisse zur Stellung von Schlichtung in einer Menge unterschiedlicher Verfahren der Konfliktbearbeitung, dem kulturellen Paradigma. Diese Ergebnisse betrefien den gesellschaftichen Stellenwert von Schlichtung, Kriterien der Schlichtungs-Wahl und der Folgen der Wahl. Als Frage, auf die diese Ergebnisse eine Antwort liefern, ließe sich formulieren: „Unter welchen Bedingungen und mit welchen Folgen wird Schlichtung als Modalität der Konfliktbearbeitung gewählt?"

- Ergebnisse zur Entwicklung und zur Karriere komplexer Verläufe der Konflikt-Bearbeitung. Diese Ergebnisse betreffen die Einbettung von Schlichtung als Moment oder Phase in einen Konflikt-Zusammenhang, das „Syntagma“. Als Frage ließe sich für diese Ergebnisse formulieren: „Wie hängt Schlichtung mit der übrigen Bearbeitung des jeweiligen Konflikts zusammen?“

- Ergebnisse, die interaktive Verlaufsmodelle für Schlichtung liefern. Schlichtung wird verstanden als kulturunabhängiges, interaktiv charakteristisches Muster der Konflikt-Bearbeitung. Die Frage, auf die diese Ergebnisse antworten, wäre: „Wie läuft Schlichtung ab?“"

- Ergebnisse zum sozial-kulturellen Bedingungszusammenhang von Schlichtung. Diese Ergebnisse nennen die Faktoren, von denen die Abwicklung und die Wirksamkeit von Schlichtung abhängt. Als Frage ließe sich formulieren: „Wieso funktioniert Schlichtung?"

Nicht in die Sichtung miteinbezogen wurden Ergebnisse zum "strukturellen Schlichten“ und zum Ansatz des ,invisible mediator“'23. Unter ,,strukturellem Schlichten“ werden soziale Strukturen verstanden, die im Falle eines Konflikts allein durch ihre Präsenz konfliktmildernd und einigungsträchtig wirksam sind. Das klassische Beispiel sind sog. „Kreuzbindungen“"24, etwa wechselseitige verwandschaftliche Beziehungen, die sich im Streitfall so auswirken, daß jede streitfortführende Aktion die eigene Verwandtschaft mitschädigen würde.

„Intergroup conflict is always moderated by the presence of members with divided loyalities ‘25.

Das interaktive Geschehen innerhalb solcher Konstellationen ist durchweg anderer Natur als der hier interessierende Gegenstand, so daß dieses Phänomen hier nicht weiter berücksichtigt wird.

Beim Ansatz des ,,invisible mediator" wird die Schlichtungs-Instanz als internalisierte Größe in den Köpfen von Gesellschaftsmitglieder betrachtet. Der Kritik von Gulli$\mathrm{ver}^{26}$, hier werde eine Überstrapazierung des Schlichtungs-Begriffs vorgenommen, ist zuzustimmen. Faktisch geht es um internalisierte Normen, bestenfalls um die kulturspezifische Konflikt-Ideologie, nicht jedoch um die Besonderheiten von Schlichtung als einem Verfahren der Konflikt-Bearbeitung.

23 Michael Barkun, Law without Sanctions. Order in Primitive Societies and the World Community, New Haven and London 1968.

24 Spittler (N. 3), 19.

25 Richard L. Abel, Theories of Litigation in Society. „Modern" Dispute Institutions in "Tribal" Society and „Tribal“ Dispute Institutions in ,Modern" Society as Alternative Legal Forms, in: Erhard Blankenburg et al. (Hrsg.), Alternative Rechtsformen und Alternativen zum Recht. Jahrbuch für Rechtssoziologie und Rechtstheorie Bd. 6, Opladen 1980, 165-191 (168).

26 Gulliver (N 4), 42. 


\section{Das kulturelle Paradigma von Verfabren der Konfliktbearbeitung}

Schlichten wird betrachtet als eines von mehreren Verfahren in einer Klasse funktional gleichwertiger Alternativen zur Bewältigung ${ }^{27}$ sozialer Konflikte mit jeweils unterschiedlichen Folgen.

Im Rahmen dieser Perspektive erfolgen Leistungszuschreibungen und -vergleich, Analysen der gesellschaftlichen Folgen der Verfahrenswahl und Untersuchungen zu den Kriterien der Verfahrenswahl selbst. Am Ausgangspunkt entsprechender Untersuchungen steht in der Regel die Aufstellung eines Paradigmas gesellschaftlicher Handlungsmuster, das als Klasse funktionaler Äquivalente für das Problem der Konfliktbewältigung betrachtet wird.

Im folgenden seien vier Paradigmen exemplarisch einander gegenübergestellt ${ }^{28}$. Deutlich wird, daß man sich in den dargestellten Kulturen offenbar ähnlicher Verfahren bedient, die in Einzelheiten, vor allem aber in ihrem paradigmatischen Zusammenhang, variieren ${ }^{29}$.

Paradigmen von gesellschaftlichen Handlungsmustern zur Konfliktbewältigung:

\begin{tabular}{l|l|}
\begin{tabular}{l|l}
$\begin{array}{l}\text { Nader \& Todd } \\
\text { (allgemein) }\end{array}$ & \multicolumn{1}{|l}{$\begin{array}{l}\text { Todd } \\
\text { (bayer. Wald) }\end{array}$} \\
\cline { 2 - 2 } $\begin{array}{l}\text { übersehen } \\
\text { aus dem Wege } \\
\text { gehen }\end{array}$ & $\begin{array}{l}\text { nicht reagieren } \\
\text { direkte Kon- } \\
\text { frontation } \\
\text { informelle } \\
\text { Schlichtungs- } \\
\text { gewalttätige } \\
\text { Lösung }\end{array}$ \\
$\begin{array}{l}\text { Aushandle (Gast- } \\
\text { zimmer) } \\
\text { formelle } \\
\text { Schlichtungs- } \\
\text { Stelle } \\
\text { Gericht }\end{array}$ \\
Schlichtung \\
Schiedsverfahren \\
Urteilsverfahren
\end{tabular} &
\end{tabular}

\begin{tabular}{l} 
Rothenberger ${ }^{32}$ \\
(sunnit. Dorf, Libanon) \\
\begin{tabular}{|l|}
\hline übersehen \\
gewalttätige Lösung \\
Aushandlung \\
Aushandlung mit \\
Hilfe eines Neutralen \\
Schlichtung \\
Antrag an Mukhtaar \\
Gericht
\end{tabular} \\
\hline
\end{tabular}

Canter ${ }^{33}$

(Sambia)

ignorieren
Selbsthilfe
private Schlichtung
öffentl. Schlichtung
örtliches Gericht
Magistratsgericht

ignorieren

27 „Bewältigung“ wird hier nicht im Sinne eines Erfolgswortes verwendet, sondern als Ausdruck zur Bezeichnung der sozialen bzw. interaktiven Auseinandersetzung mit dem Konflikt.

28 Ähnlich unterscheidet auch Spittler (N. 3):
1 Selbsthilfe,
2. Verhandlung,
3. Vermittlung,
4. Entscheidung (Schiedsspruch),
5 Rechtsorganisation.

Spittler, Konfliktaustragung in akephalen Gesellschaften: Selbsthilfe und Verhandlung, in: Blankenburg et al. (Hrsg.) (N. 25), 142-164 gibt einen interessanten Hinweis auf die Funktionsweise von ,Selbsthilfe': diese muß, damit die dysfunktionalen Folgen nicht überwiegen, durch Fortsetzung der Fußnoten 28-33 siehe Seite 38 
Uber die Angabe der kulturspezifischen Paradigmen hinaus besteht ein weiteres Resultat entsprechender Arbeiten in der Ermittlung sozial-kultureller Bedingungen, die im Streitfall die Selektion eines der Verfahren steuern.

Canter $^{34}$ z. B. gibt die Umstände und Voraussetzungen an, unter denen sich für die Lenje, einen Stamm in Sambia, die Präferenz eines Verfahrens vor den anderen ergibt:

- Das Ignorieren einer Schädigung erweist sich als funktional, wenn der Geschädigte das Risiko hoher Streitkosten vermeiden will oder von der kulturellen Regel Gebrauch machen will, Schädigungen ,,aufzusparen" und später kumuliert abzurechnen (z. B. bei Ehestreitigkeiten),

- Die Entscheidung für eine private Schlichtung ist indiziert, wenn es der Partei darum geht, das Hochschaukeln von Emotionen zu vermeiden und für sie der Umgang mit Problemen des Gesichtsverlusts im Vordergrund der Konfliktbearbeitung steht.

- Die öffentliche Schlichtung wird bei bestimmten Typen von Streitfällen gewählt, insbesondere dann, wenn die ganze Familie von dem Streit betroffen ist.

- Das Gericht wird angerufen, wenn die Orientierung, Recht zu behalten, im Vordergrund des Streits steht.

Rothenberger ${ }^{35}$ geht auf die besondere Leistungsfähigkeit des Schlichtungs-Verfahrens in einer sunnitischen Gemeinde im Libanon ein: die Vorteile einer Konfliktbearbeitung durch Schlichten liegen vor allem darin, daß die sozialen Beziehungen zwischen den Streitparteien relativ gering belastet werden, daß die für die Beteiligten mit dem Streit verbundenen Relevanzen (Ehre, Stolz, etc.) ohnehin nicht gerichtsfähig sind und daß durch die Uberführung eines Streites in einen Sippenstreit die Eskalationsgefahr bedrohlich wachsen würde.

Todd $^{36}$ macht darauf aufmerksam, daß die Funktionalität einzelner Paradigma-Elemente nicht nur auf den Streitfall bezogen sein muß, sondern auch in übergeordneten sozialen Bezugsgesichtspunkten liegen kann. Er zeigt, daß die Schlichtungsstelle in einem Dorf im Bayerischen Wald für die Außenseiter der Gemeinde (, Taugenichtse") eine Möglichkeit der Streitaustragung mit dem Dorf-Etablishment darstellt, mit der sie zugleich den Anspruch erheben können, ebenfalls zum Establishment zu gehören. Die Auswahl eines Verfahrens wird also gesteuert von Gesichtspunkten der Statuserhöhung.

Fortsetzung Fußnote 28

ein Netz intensiver Kommunikationsverhältnisse abgefedert werden, wobei die Kommunikationsereignisse selbst nicht ergebnisorientiert auf Konfliktlösung zu sein brauchen.

29 Die Reihenfolge, in der die Verfahren im Paradigma stehen, gibt nicht die Reihenfolge an, in der sie im Verlauf einer Konflikt-Bewältigung abgewählt werden können. Die einzelnen Paradigmen sind intern unterschiedlich gegliedert. Unmittelbare Korrespondenzen zwischen verschiedenen Paradigmen bestehen nicht.

30 Nader/Todd (N. 18).

31 Harry $F$ Todd, Litigous Marginals: Character and Disputing in a Bavarian Village, in: Nader/ Todd (eds.) (N. 18), 86-121.

32 John E. Rothenberger, The Social Dynamics of Dispute Settlement in a Sunni Muslim Village in Lebanon, in: Nader/Todd (eds.) (N. 18), $152-180$.

33 Richard S. Canter, Dispute Settlement and Dispute Processing in Zambia. Individual Choice versus Societal Constraints, in: Nader/Todd (eds.) (N 18), 247-280.

34 Canter (N. 33), $257 \mathrm{ff}$.

35 Rothenberger (N. 32).

36 Todd (N 31). 
,the agency has provided a convenient forum for marginals in which claims to character could be lodged and status fought for It has provided an arena for institutionalized ,prestige contests, " and a procedure that allows the Gottfrieding marginals to establish a pecking order among and between themselves, as well as to try to establish a status claim vis-à-vis village insiders. " 37

Eine dritte Gruppe von Ergebnissen betrifft die gesellschaftlichen Folgen der Verfahrenswahl und des erhobenen Paradigmas insgesamt. So zeigt sich in verschiedenen Untersuchungen, daß bestehende Machtverhältnisse durch das Funktionieren des Paradigmas stabilisiert werden und sich soziale Ungleichheiten perpetuieren $\operatorname{Todd}^{\mathbf{3 8}}$ zeigt, daß die oben angegebenen ,,marginals“ in einem bayerischen Dorf zu der von den ,insidern" akzeptierten Schlichtungs-Arena, dem Gastzimmer, keinen Zugang haben und daher für Konfliktregelungen auf überörtliche Instanzen angewiesen sind. Zugleich gilt in dem Dorf jedoch die Ideologie der gemeindeinternen Konfliktaustragung mit Präferenz für Schlichten. Die "marginals" geraten in eine Zwickmühle (Todd spricht von ,double bind“): sie können ihre Ansprüche in Streitfällen nur durchsetzen, indem sie gegen etablierte Normen verstoßen und perpetuieren damit ihren sozialen Status als "marginals". (Hinzu kommt die Gefahr, daß sie qua gerichtlichem Verfahren kriminalisiert werden und so ihre Außenseiterrolle noch verstärkt wird).

Canter $^{39}$ zeigt in einer vergleichbaren Untersuchung, daß die Lenje in Gebieten, in denen sie Majorität besitzt, Nicht-Lenje vom Zugang zu den informellen Verfahren ausschließen bzw. diese Verfahren von den Nicht-Lenje aus Mißtrauen nicht in Anspruch genommen werden. Über differentiellen Zugang zu den Verfahren wird die Integration innerhalb der Lenje somit gestärkt, die Abgeschlossenheit nach außen erhöht und die Machtverhältnisse insgesamt werden stabilisiert.

\section{Das Syntagma der Bearbeitungsgescbicbte eines Konflikts}

Die paradigmatische Perspektive betrachtet das Arsenal gesellschaftlich aufbereiteter und - differentiell - zur Verfügung stehender Verfahren der Konfliktbearbeitung. Die syntagmatische Perspektive fokussiert auf den komplexen Prozeß der Bearbeitung eines Konfliktfalls insgesamt ${ }^{40}$ Untersuchungsgegenstand ist der gesamte Konfliktfall in seiner zeitlichen, sachlichen und sozialen Komplexität. Schlichtung wird betrachtet als ein Ereignis in dem gesamten Prozeß, das in seiner Position interpretiert und in seiner Wirkung auf die weitere Geschichte bestimmt wird.

Colson $^{41}$ führt in ihrer klassischen Arbeit über einen Volksstamm in Sambia diesen Ansatz exemplarisch vor: an einem Mordfall zeigt sie, wie Gesellschaftsmitglieder die einschlägigen Regeln und Maximen handhaben und interpretieren, wie Verwandt-

37 Ebd., 111.

38 Todd (N. 31$)$.

39 Canter (N. 33).

40 Nader/Todd (N. 18), 15-30.

41 E. Colson, Social Control and Vengeance in Plateau Tonga Society, in: Africa 23 (1953), 188212. 
schaftsbeziehungen einer Eskalationsgefahr in der Konfliktbearbeitung entgegenwirken und welche Strategien die einzelnen Parteien verfolgen. Gulliver ${ }^{42}$ beschreibt anhand von fünf Konfliktfällen, wie die Streitregelung in einem Fall die Konfliktbewältigung bei nachfolgenden Streitigkeiten innerhalb einer engeren Gemeinschaft beeinflußt.

Nader \& Todd $^{43}$ vertreten einen solchen Ansatz programmatisch. Er ist gekennzeichnet durch eine Betonung des sozialen Umfeldes, in das die Verfahren der Konfliktbearbeitung eingebettet sind (gegenüber einer Fokussierung auf die Verfahren selbst) und durch die Betonung der Perspektiven und Interessen der einzelnen Beteiligten (gegenüber einer Fokussierung auf die verfahrenswaltenden Institutionen und ihre Zwecksetzungen). Entsprechend ergibt sich ein gegenüber dem paradigmatischen Ansatz unterschiedliches Profil von Untersuchungsvariablen; wichtig werden vor allem:

- die Sozialbeziehungen zwischen den Streitparteien und ihr Einfluß auf die Konfliktgeschichte (Lohnabhängigkeit, Freundschaft) ${ }^{44}$;

- die Beziehungen zwischen den Streitparteien und ihrem jeweiligen sozialen Umfeld und dessen Einfluß auf die Konfliktgeschichte (Verwandtschaft, Nachbarschaft, aber auch insider-outsider $^{45}$;

- die Typen von Gegenständen (materielle Schädigung, Normverletzung, Streit um knappe Ressourcen), ihre kulturspezifische Interpretation und deren Auswirkung auf den Konfliktverlauf (so wird z. B. unter sardischen Schäfern der Viehdiebstahl nicht notwendig als Verbrechen wahrgenommen: ,if somebody steals my flock, he does not offend me. It depends, depending on who he is, he offends me, and how he steals, and why .... ${ }^{446}$;

- die Ziele und Strategien, die die Streitparteien in der Konfliktbearbeitung verfolgen und deren Änderung die Konfliktgeschichte mit beeinflußt;

- die kulturspezifischen Werte, die mit Streitfällen verknüpft sind, und ihr Einfluß auf den Prozeß der Konfliktbearbeitung (Familienloyalität, Verpflichtungen, Schamgefühl, Emotionen) ${ }^{47}$;

- die Zeitspanne zwischen Zeitpunkt der Schädigung und Konfliktbearbeitung in ihrer kulturdifferentiellen Bedeutung (entweder verhärten sich die Fronten oder der Konflikt verliert an $\mathrm{Be}$ deutung);

- die kulturspezifische Streit-Ideologie, d. h. die Einstellung Konflikt und Streit gegenüber; in einigen Kulturen ist Konflikt normaler Bestandteil des Alltagslebens (z. B. Tswana) ${ }^{48}$, in anderen herrscht extreme Empfindlichkeit schon gegenüber leisen Mißstimmigkeiten ${ }^{49}$;

- die kulturspezifische Bewertung von Identitätsmerkmalen wie ,Urteilsfähigkeit', ,Charakter ${ }^{50}$; ,Empfindlichkeit', ,Stolz'; „Ehre', ,Mut' ${ }^{51}$ durch die der Konflikt eine komplexe Dimensionierung erfährt;

- die Zugänglichkeit der einzelnen Verfahren der Konfliktbearbeitung (s.o.).

42 P. H. Gulliver, Dispute Settlement without Courts: The Ndendeuli of Southern Tanzania, in: Nader (ed.), Law in Culture and Society, Chicago 1969, 24-68.

43 Nader/Todd (N. 18).

44 Siehe auch Gulliver (N. 42).

45 Siehe auch Gulliver (N. 42).

46 Brigaglia, zitiert nach Nader/Todd (N. 18), 34.

47 Vgl. Simon Roberts, Ordnung und Konflikt. Eine Einführung in die Rechtsethnologie, Stuttgart 1981, 55.

48 Vgl. Comaroff/Roberts (N 7).

49 Vgl. A. L. Kroeber, Zuni Kin and Clan, New York 1917.

50 Todd (N. 31).

51 Rothenberger ( $N$ 32). 
Nader \& Todd $^{52}$ unterscheiden drei Stadien, in die sich ein Konfliktfall heuristisch gliedern läßt:

- "grievance stage", das sind Umstände, die eine Person/Partei als Schädigung oder Unrecht wahrnimmt und die die Beschuldigung (,,complaint") begründen;

- ,conflict stage“, das sind die Aktivitäten, die im Zuge der Wendung in der Sache an einen Anderen - die andere Partei - erfolgen, also der Streit im engeren Sinne;

- „dispute stage“, das ist die Veröffentlichung des Konflikts über die zwei Parteien hinaus. In dieser Phase können die meisten der oben beschriebenen Verfahren der Konfliktbearbeitung aktualisiert werden.

Die einzelnen Stadien sind an diese Reihenfolge nicht notwendig gebunden; ein Geschädigter kann sich z. B. gleich an die Öffentlichkeit wenden; ein Konflikt kann nach öffentlicher Behandlung wieder auf bilateralem Niveau weiterbehandelt werden. Die Phasen stellen aber Epochen in der Konfliktgeschichte mit je besonderen Verlaufscharakteristika und typischen Resultaten dar.

Einen zu Nader \& Todd vergleichbaren Ansatz entwickeln Comaroff \& Roberts ${ }^{\mathbf{5 3}}$ für Tswana. Als ihr Untersuchungsziel bestimmen sie, ,the logic of processes and their location within the socio-cultural orders in which they occur". Untersuchungsgegenstand ist wie bei Nader \& Todd der jeweilige Konflikt in seiner komplexen Verlaufsgestalt; untersucht werden die Variablen, denen die je besondere Gestalt geschuldet ist. Eine Kernvariable, die Comaroff $\&$ Roberts $^{54}$ herausarbeiten, läßt sich als Inhalts-Beziehungsprofil des Streitgegenstandes kennzeichnen: je nach Ausprägung des Gegenstandes auf einer Dimension der Beziehungsspezifizität der Parteien und der Sachverhalts-Spezifizität des Gegenstandes ergeben sich unterschiedliche Konflikttypen, mit denen die Parameter der Konfliktbearbeitung variieren, wie folgender Uberblick zeigt:

Insbesondere die Bestimmung des Konfliktgegenstandes ist von übergeordneten Zwecksetzungen und Interessen der Parteien gesteuert; sind sie z. B. in Geldnot, werden sie den Gegenstand so bestimmen, daß ein rasches Verfahren möglich ist; ist eine Partei am Ausbau ihrer Karriere interessiert, wird sie den Gegenstand als diffus präsentieren, weil ihr dies den Zugang zu einem öffentlichen Verfahren ermöglicht (und damit ein Auditorium für das eigene rhetorische Talent besorgt ist). Grundsätzlich liegt für die Tswana die mit Streiten verbundene Ideologie auf Gewinnen, nicht auf Lösen des Konflikts. Die Frage des Verfahrenszugangs ist wesentlich, weil in der Regel jede der Parteien in verschiedenen Instanzen ,ihre Leute" sitzen hat. Der weitere Bearbeitungsweg ist - vor allem durch Definition des Konfliktsachverhalts - Gegenstand des Bearbeitungsprozesses selbst und befindet sich damit in partieller Kontrolle der Parteien selbst. 


\begin{tabular}{|c|c|c|c|c|c|}
\hline $\begin{array}{l}\text { Parameter der } \\
\text { Konfliktbearbeitung }\end{array}$ & $\begin{array}{l}\text { Inhalts- } \\
\text { Beziehungs- } \\
\text { profil }\end{array}$ & $\begin{array}{c}\text { Typ 1 } \\
\text { klar definierter } \\
\text { Konfliktgegenstand } \\
\text { ohne Vorgeschichte }\end{array}$ & $\begin{array}{c}\text { Typ II } \\
\text { klar definierter } \\
\text { Konfliktgegenstand; } \\
\text { Parteien besitzen über- } \\
\text { dauernde Beziehungs- } \\
\text { struktur }\end{array}$ & $\begin{array}{c}\text { Typ III } \\
\text { Konfliktgegenstand } \\
\text { nicht eindeutig bestimm- } \\
\text { bar; keine überdauernde } \\
\text { Beziehung }\end{array}$ & $\begin{array}{c}\text { Typ IV } \\
\text { Konfliktgegenstand } \\
\text { nicht eindeutig bestimm- } \\
\text { bar; überdauernde } \\
\text { Beziehung }\end{array}$ \\
\hline
\end{tabular}

Tendenz zunehmend

Flexibilität des Verfahrenszugangs und einsatzes (bilateral $\rightarrow$ Suche nach informellen Dritten $\rightarrow$ Wendung an Häuptling $\rightarrow$ Anrufung des Richters)

Einbezug aktueller Situationsmerkmale

\section{Inkorporation von Vorgeschichte}

Bestimmtheit des Streitobjekts
Tendenz abnehmend restriktiv; Mißachtungen werden negativ sanktioniert

repräsentieren

Atiologie des Konflikts

\section{Tenden zunehmend}

Splelraum bei
Instanzenwahl

\section{Tendenz abnehmend}

Tendenz zunehmend

entfällt konkurrierende Wirklichkeits-Konstruktion 


\section{Prozeß-Modelle für Schlichtungs-Interaktion}

Für beide bisher dargestellten Ansätze gilt, daß für sie die interaktive Dimension von Schlichtungsprozessen keine besondere Rolle spielt. Dieser Befund ist aus der Perspektive der Ansätze selbst leicht plausibel zu machen:

In paradigmatischer Perspektive interessiert die Einbindung von Verfahren der Konfliktbearbeitung in den sozial-strukturellen Zusammenhang, ihre Leistungsfähigkeit dort und ihre Dysfunktionen. Der Verfahrenscharakter selbst und ein bestimmter Typ von Resultat ist idealtypisch vorausgesetzt; die ,interne“ interaktive Dynamik, durch die das Verfahren situativ hergestellt wird, kann bei dieser Fragestellung außer acht bleiben.

In syntagmatischer Perspektive interessiert die komplexe Verlaufsgestalt der Konfliktbearbeitung insgesamt, die mit Hilfe der Sichtweise der Beteiligten und Merkmalen des kulturellen Wert- und Ideologiesystems rekonstruiert wird. Die einzelnen Interaktionsprozesse fallen in dieser Perspektive durch: die komplexe Verlaufsgestalt geht von der Gegebenheit des Verfahrenscharakters der Schritte bereits aus - wenn dieser überhaupt interessiert - die Rekonstruktion der Verlaufsgestalt erfolgt gerade nicht aus interaktiver Perspektive, sondern durch Komposition der Perspektiven der einzelnen Teilnehmer.

Für eine gesprächsanalytische Orientierung auf Schlichten ist es freilich von hohem Interesse, gerade Ergebnisse zu erfahren, die quasi ,zwischen“ diesen beiden Ansätzen liegen. Offenbar sieht man auch in der Ethnologie selbst das entstandene Desiderat, die interaktive Dimension der einzelnen Formen der Konfliktbearbeitung begrifflich zu bestimmen und empirisch zu erfassen ${ }^{55}$.

Für Schlichten finden sich zwei solche Ansätze, die im folgenden genauer vorgestellt werden sollen: Der eine Ansatz ist ein Vorschlag von Nader ${ }^{56}$, der darauf hinausläuft, Schlichten als einen besonderen interaktiven Stil der Konfliktbearbeitung zu begreifen und diesen Stil über ein Bündel von Verhaltensmerkmalen zu bestimmen: Der andere Ansatz ist ein Versuch von Gulliver ${ }^{57}$, Schlichten als spezifische Variante von Aushandlung zu betrachten und anzugeben, wie sich diese Variante in einem Phasenmodell von Aushandlung darstellt.

Nader ${ }^{58}$ betrachtet den interaktiven Stil bei der Konflikt-Bearbeitung als Schlüsselvariable rechtssoziologischer Forschung. Eine Typologie von Bearbeitungsstilen sei anderen typologischen Entwürfen (nach Sanktionstyp, Komplexität des Rechts, Verfahrensresultat) vorzuziehen. In einer Untersuchung der Konfliktbearbeitung bei den Zapotec in Mexiko stellt Nader fest, daß dort eine ausgeprägte Streit-Ideologie der Herstellung von Balance besteht und dementsprechend Streit zunächst auf gütlichem Wege

55 Marjorie H. Goodwin, Processes of Dispute Management among Urban Black Children, in: American Ethnologist 9 (1982), 76-93.

56 Laura Nader, Styles of Court Procedure: To Make the Balance, in: Laura Nader (ed.), Law in Culture and Society, Chicago 1969, 69-91.

57 P H. Gulliver, Negotiations as a Mode of Dispute Settlement: Towards a General Model, in: Law and Society Review 7 (1973), 667-691; Gulliver (N. 4).

58 Nader (N. 56). 
unter Konzession beider Seiten beizulegen versucht wird. Das anhand von Fallbeispielen illustrierte Muster läßt sich als Schlichtung gemäß unserer Arbeitsdefinition kennzeichnen.

Nader bestimmt dieses Muster als „Stil“. Stil ist definiert als Bündel derjenigen Interaktionsmerkmale der beobachteten Fälle, die über alle Fälle konstant auftreten. Es sind dies folgende Merkmale:

1. Die Aktivitäten des Schlichters zielen darauf ab, den Grund des Streits zu ermitteln.

2. Ziel der Konfliktbearbeitung ist es, die Differenzen der Parteien zu verringern (Minimax-Prinzip).

3. Bei der Lösungssuche werden die sozialen Netzwerke, in denen sich die Parteien befinden, mitberücksichtigt.

4. Normen und Interessen werden in Rechnung gestellt.

5. Der Schlichter ist ein Rechtsexperte.

6. Die Definition vergangener Tatbestände oder Schuldzuschreibung ist nicht erforderlich.

7. Die Lösung hat die Form einer Vereinbarung.

8. Die Vereinbarung wird durch Zwangsmittel gestützt.

9. Die im Verlauf der Bearbeitung angestellten Oberlegungen und Erwägungen sind auf die Zukunft gerichtet.

10. Positionen der Streitparteien werden im Verlauf des Geschehens austauschbar.

11. Es besteht eine Präferenz für Kompromisse auch dann, wenn eine Entweder-Oder-Entscheidung möglich wäre.

Dieses Merkmalsbündel bestimmt Schlichten, und zwar - wie Nader betont - unabhängig von den jeweiligen formalen Situationsdefinitionen, dem kulturellen und organisatorischen Kontext und dem jeweiligen gesellschaftlichen (ökonomischen und politischen) Entwicklungsstand, in dem es auftritt.

Die „kulturelle Komponente“ beeinflußt nicht die interaktiven Merkmale des Stils der Konfliktbearbeitung, sondern setzt höher an bei der Selektion eines Stils aus einem Paradigma möglicher Stile. Die Selektion von Schlichtung als Stil der Konflikt-Bearbeitung erfolgt insbesondere dann,

1. wenn die kulturelle Streit-Ideologie auf Ausgleich orientiert ist,

2. wenn die Beziehungen zwischen den Streitparteien aufrecht erhalten werden müssen,

3. wenn bestimmte Typen von Streit-Gegenständen vorliegen.

Naders Ansatz kann als ein erster Versuch gewertet werden, Schlichtung in seiner kommunikativen Besonderheit zu bestimmen und mit Interaktionskategorien zu benennen, ohne zentral auf sozial-strukturelle Kategorien zurückzugreifen. Naders Merkmals-Cluster scheint für eine Definition eines bestimmten Typs von Schlichtung - den im rechtlichen Bereich stattfindendenden - durchaus geeignet. Als Instrument für gesprächsanalytische empirische Studien ist das Cluster allerdings nicht geeignet, da es keine Verlaufsmerkmale enthält, sondern Merkmale, die nur dem abgelaufenen SchlichtungsProzeß insgesamt zugeordnet werden können.

Einen Schritt weiter geht Gulliver ${ }^{59}$, der ein Prozeßmodell für Schlichtung entwickelt. Gulliver gewinnt dieses Modell interessanterweise aus einem allgemeinen interaktiven 
Verlaufsmodell für bilaterale Aushandlung (negotiation), für das er Kulturunabhängigkeit beansprucht. Er betont die Identität der Handlungsziele von Aushandlung und Schlichtung als Herstellung von Einigung zwischen den Parteien (im Gegensatz zu Verfahren der Entscheidung eines Dritten). Gulliver begreift Schlichtung als eine besondere Aushandlungsvariante, in der das Aushandlungsziel durch die Intervention eines Dritten, des Schlichters, erreicht wird $(, \ldots$. a facilitator in the process of trying to reach agreement ${ }^{\text {(60 }}$ ). Gulliver sieht die Leistung der Intervention eines Dritten im Aushandlungsprozeß u. a. darin, die Übergänge zwischen den verschiedenen Phasen (s. u.) zu erleichtern und bewerkstelligen zu können, die Orientierungen der Parteien zu steuern und zu verändern, Fokussierungen auf den Stand der Verhandlungsprogression vorzunehmen und Erwartungen auszurichten und zu korrigieren.

Die Erweiterung des bilateralen Aushandlungsmodells um einen Dritten hat zwei Konsequenzen für die Modellbildung:

- die Phasen des Aushandlungsprozesses werden differenzierter,

- das Modell wird um eine Bestimmung der verschiedenen möglichen Schlichtungsrollen bzw. -strategien ergänzt.

Gulliver nimmt für sein Modell eine Sequenz überlappender Phasen an, wobei jede Phase in einer "progression towards settlement ${ }^{\text {"61 }}$ den Vollzug der nächsten Phase vorbcreitet; die Phasen stehen also in einem Voraussetzungsverhältnis zueinander. Die Phasen unterscheiden sich hinsichtlich des Inhalts der ausgetauschten Information, der assoziierten Lernprozesse und des erforderlichen Ausmaßes an Koordinationsarbeit.

Die Übergänge erfolgen in Schwellen (,,accumulative trends“62 ${ }^{\text {(62 }}$. Gulliver ${ }^{63}$ unterscheidet eine Vorphase von Schlichtung, in der die Publizierung des bilateralen Konfliktes und die Wahl der Schlichtungs-Instanz (,,arena") erfolgt, vom eigentlichen Schlichtungsverlauf, der in folgende Phasen gegliedert ist:

1. Phase: Definition des Gegenstands. In dieser Phase erfolgt die Präsentation der jeweiligen Konflikt-Versionen, hier werden die Versionen wechselseitig wahrgenommen und beurteilt (was der andere weiß, wie er sein Wissen konstruiert, was er betont, welche Strategie er verfolgt). Die Funktion dieser Phase im Hinblick auf das Handlungsziel des Schlichtungsmusters ist klar: die Situationsdefinition gibt vor, was behandelt werden muß. Gulliver trifft eine Unterscheidung zwischen der manifesten Streitursache (,overt cause") und dem tatsächlichen Streitpunkt (,real matter") ${ }^{64}$.

2 Pbase: Herausarbeiten der parteispezifiscben Differenzen am identiscben Gegenstand. In dieser Phase sind folgende Aktiviläten dominant: ,initial stands deciared, opening bits made, ideologies aired, strengths paraded, weaknesses expounded“ "65. Durch das Herausarbeiten der Differenzen umgrenzen dic Parteien das Handlungsfeld und präzisieren dessen Binnenstruktur.

3. Pbase: Einigungssuche. Hier erfolgt der Wechsel der Orientierung von Differenzen hin zu Koordination und Kooperation; die Angleichung der Differenzen erfolgt durch Ausloten eines akzeptablen Einigungsbereichs und Herauslösen akzeptabler und negibler Punkte.

4. Phase: Ritualisierung der Einigung.

60 Gulliver (N 4), 15.

61 Gulliver (N 4), 22.

62 Gulliver (N. 4), 24.

63 Gulliver (N 57), 676-680.

64 Gulliver (N. 57), 676.

65 Gulliver (N. 4), 23. 
General Model of the Process of Negotiations ${ }^{66}$

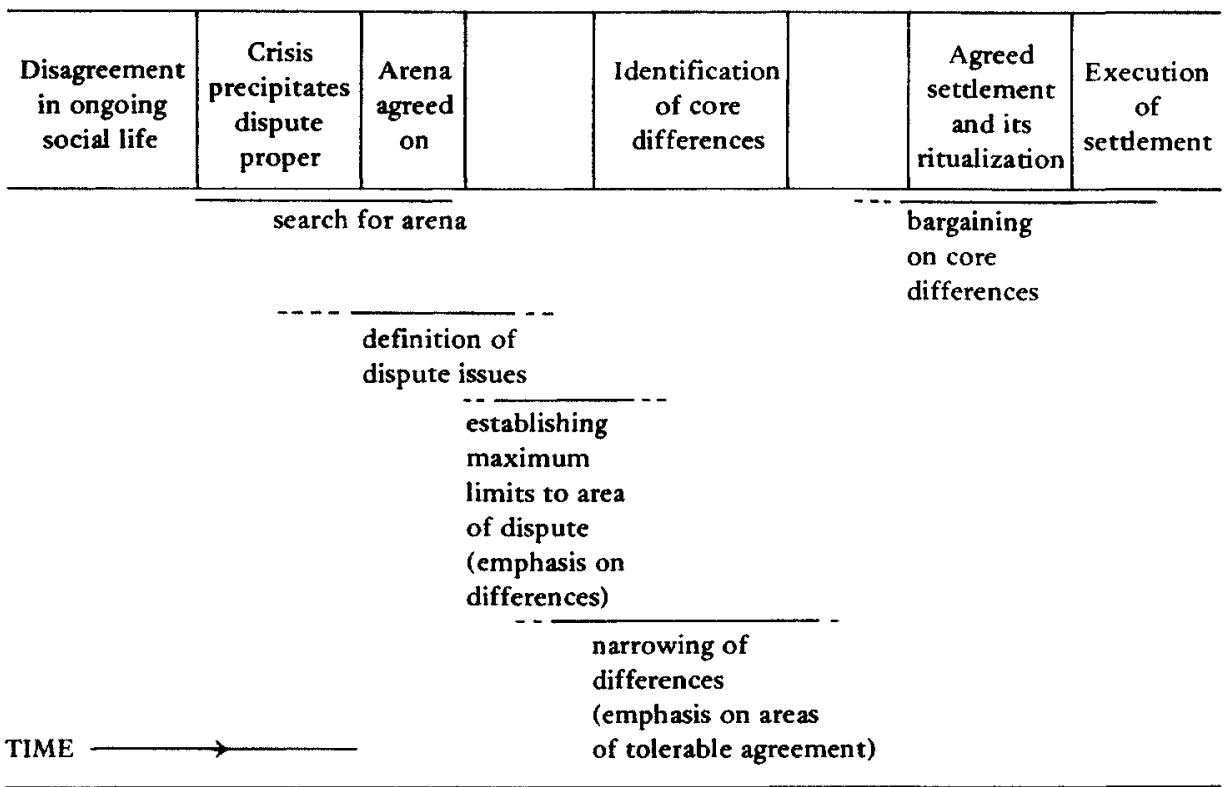

The process may be stopped at any point in frustrated disagreement, and it may be restarted at some earlier point, perhaps with changed teams and a different arena. Time lengths of successive phases vary proportionally to each other in different cases. There may be several sessions of negotiations in the total process, with a minimum of one to determine the arena, and one thereafter in that arena.

Dieses Modell gilt für bilaterale Aushandlungen wie für Schlichtungs-Interaktion gleichermaßen. Der Schlichter wird zu dem bilateralen Modell gleichsam nachträglich hinzukomponiert: Er will in dieser Struktur operieren und muß sich ihr anpassen. Erfolgt diese Anpassung nicht, kommt es zu Turbulenzen und Fehlentwicklungen, z. B. wenn der Schlichter in der Phase der Verdeutlichung der Differenzen sich bereits um eine Einigung bemüht. Gulliver ${ }^{67}$ sieht 7 Varianten der Intervention eines Dritten in die skizzierte Aushandlungsstruktur, sog. Schlichtungs-Strategien, die sich in ihrer Interventionsstärke unterscheiden. Die Abwahl der Varianten wird von sozial-strukturellen und kulturellen Bedingungen gesteuert. Die Strategien sind:

- Der Effekt ,reiner" Ko-Präsenz liegt vor allem darin, daß durch die Anwesenheit eines Dritten der Umgang zwischen den Streitparteien verbessert und der Legitimationsdruck für die eigene Position erhöht wird. Gulliver nennt als Beispiele für diese Strategien den amerikanischen Industrie-Schlichter. Diese Variante ist indiziert, wenn sich bilaterale Verhandlungen festgefahren haben oder zwischen den Parteien persönliche Feindschaften bestehen. 
- Der ,,chairman" greift nicht in die inhal tliche Auseinandersetzungen ein, sondern regelt nur den Vollzug der Auseinandersetzung selbst. Der Effekt dieser Strategie liegt wesentich darin, die Interaktion vor dem Zusammenbruch zu bewahren.

- Der Fokussierer bündelt Phasen des Aushandlungsverlaufs inhaltich und sichert erzielte Ergebnisse ab.

- Der Werte-Verwalter reguliert den Verhandlungsprozeßs bereits inhaltlich durch das Einspeisen von Positions-Beurteilungen, die aus allgemeinen gesellschaftlichen Normen und Standards abgeleitet sind.

- Der Experte (,enunciator") steckt die Grenzen des Verhandl ungsspielraums ab und eröffnet Verhandlungsmöglichkeiten.

- Der Verfabrenswalter klärt Angaben und Informationen, ermuntert zur Einigung, rephrasiert Positionen, klärt Mißverständnisse, achtet auf Thematizität der Beiträge und fokussiert auf relevante Punkte. Diese Schlichtungs-Strategie ist besonders innerhalb der Phase 3 indiziert.

- Der Fibrer (,,leader") bringt seine eigenen Positionen ein und bewertet die Positionen der Parteien. Indiziert ist diese Strategie u. a. dann, wenn Parteien Positionen aufgeben wollen, dies aber von sich aus nicht tun können (z. B. aus Gründen des Gesichtsverlusts).

Außerdem gibt es den Sonderfall des ,go between": Dieser hebt die unmittelbare face-to-face-Situation auf; er übermittelt (und selegiert und verändert) Mitteilungen zwischen den beiden Streitparteien (z. B. der Monkalun bei den Ifugao).

In welcher Variante auch immer der Schlichter in dem bilateralen Aushandlungsprozeß agiert, hervorstechendes Merkmal seiner Rolle ist seine Neutralität. Gulliver macht aber zugleich darauf aufmerksam, daß faktisch oft vielfältige Eigeninteressen des Schlichters in das Schlichtungsgeschehen miteingehen. Die Vorstellung des neutralen Schlichter ist - so Gulliver - ein Stereotyp der westlichen Kultur, das weder kulturübergreifend, aber auch noch nicht einmal für den eigenen Kulturkreis zutrifft (ein ,,ethnocentric bias" “68). Nur allzu evident ist, daß es oft Eigeninteressen des Dritten sind, die diesen zur Übernahme der Schlichterrolle motivieren. Aus Gründen, die im Verfahren selbst zu liegen scheinen, ist es aber offenbar für die Teilnehmer erforderlich, daß sie - quasi in einer kontrafaktischen Unterstellung - von diesen Eigeninteressen des Schlichters abstrahieren (als analytische Forschungsmaxime freilich empfiehlt Gulliver, das Schlichtungs-Handeln als interessengebundenes Handeln zu analysieren); wo dies nicht mehr gelingt, wird die Durchsetzung des Eigeninteresses des Schlichters der Kostenseite des Verfahrens zugerechnet und akzeptiert. Gulliver ${ }^{69}$ unterscheidet 4 unterschiedliche Interessenprofile, die ein Schlichter haben kann:

- den ,interessenlosen Scblichter', der keine Beziehungen zu den Streitparteien besitzt und dessen Relevanzsystem von den Streitparteien nicht tangiert wird, z. B. ein religiöser Führer eines Stammes, der ,big man“ bei den Ifugao, ein Fremder, ein Experte oder auch der institutionalisierte Schlichter (Schiedsmann, leopard skin-chief (Nuer), Pathan Saint (Waigali));

- den ,interessierten Schlichter', den der Streit zwischen 2 Parteien unmittelbar in Mitleidenschaft zieht, z. B. ein Nachbarland von Staaten, zwischen denen Krieg droht; ein Pächter eines Stücks Land, um dessen Besitz sich zwei Gutsherren streiten;

- den ,parteilichen Scblichter", wenn dieser auch of t nur als letzte Instanz angerufen wird. Gulliver verweist auf Fälle, in denen offenbar trotz der Parteinahme für einen der Teilnehmer ein ,Drit- 
ter" $^{\text {“ }}$ die Schlichter-Rolle ausfüllen konnte ${ }^{70}$. Lapidar Gulliver: „Sometimes anyone is better than no one at all" 71 ;

- den Repräsentanten der Ordnung, dessen Interessen mehr auf die Aufrechterhal tung allgemeiner Werte und Ordnung gerichtet sind und nicht so sehr auf den konkreten Streitfall selbst; Starr \& Yngvesson 72 sprechen von der Bewahrung des ,,status quo"

Wie der Schlichtungs-Verlauf durch diese unterschiedlichen Interessenprofile systematisch beeinflußt wird, ist freilich noch Forschungs-Desiderat.

\section{Das Operieren von Scblichten im kulturspezifiscben Bedingungszusammenbang}

Gullivers Modell kommt von allen Beiträgen aus dem Bereich der Rechts-Anthropologie Vorstellungen einer gesprächsanalytisch orientierten Untersuchung von Schlichtungs-Interaktionen zweifelsohne am nächsten. Darin liegt der hohe Anregungs- und Herausforderungsgehalt dieses Konzeptes für die eigene Modellbildung. Freilich birgt die Konzeptnähe des Gulliverschen Ansatzes auch ein Defizit, das durch Sichtung der rechtsanthropologischen Arbeiten gerade behoben werden sollte: Die Erwartung war ja, durch die Literatursichtung etwas über die kulturelle Varietät von SchlichtungsProzessen und die Bedingungen ihres Funktionierens zu erfahren. Der Gulliversche Ansatz ist jedoch schon zu ,interaktionsnah", als daß es ihm noch gelingen könnte, die Einbettung des Schlichtungs-Geschehens in den jeweiligen kulturellen Kontext mitzuberücksichtigen. Dies dürfte freilich auch nicht in der Weise geschehen, in der es im syntagmatischen bzw. paradigmatischen Ansatz erfolgte, sondern müßte spezifischer auf die einzelnen Komponenten des Schlichtungs-Verfahrens selbst bezogen sein. Eine solche Einbettung des Schlichtungs-Geschehens in ihren jeweiligen kulturellen Kontext in sog. small-scale-Gesellschaften (kleinen, einfach strukturierten Gemeinschaften) erfolgt bei Merry ${ }^{73}$. Merry vermag vor allem zu zeigen, daß das Funktionieren von Schlichten ganz zentral von der Einbettung in einen flankierenden kulturellen und sozialen Kontext abhängt und die Ergebnisse von Schlichtungs-Prozessen wesentlich solchen Kontext-Einflüssen geschuldet sind.

Merry untersucht 5 Merkmale von Schlichtungs-Interaktionen auf ihre kulturelle Einbettung hin:

a) die Rolle des Schlichters,

b) die Entscheidung für ein Schlichtungs-Verfahren,

c) den mit Schlichtung verbundenen Zwangscharakter,

70 P. H. Gulliver, Neighbours and Networks, Berkeley 1971, 145; R. Antoun, Arab Village, Bloomington 1972, $78 \mathrm{ff}$.; R. Liebes, Contributions of Mediation to the Development of Mature Collective Bargaining, in: Labour Law Journal 9 (1958), 797-800 (798 f.).

71 Gulliver (N. 4), 36 .

72 Starr/Yngvesson (N. 11), 559.

73 Sally Engle Merry, The Social Organization of Mediation in Non-industrial Societies: Implications for Informal Community Justice in America, in: R. L. Abel (ed.), The Politics of Informal Justice, vol. 2, London 1982, 17-45. 
d) die Qualität des Resultats,

e) den Schlichtungs-Prozeß.

a) Die Rolle des Schlichters

Nicht jedes Mitglied kann in jeder Gemeinschaft die Schlichter-Rolle übernehmen; die Übernahme ist an bestimmte Qualifikationen gebunden, durch die das Einwirken sozialer Strukturen und Kräfte auf den Schlichtungs-Prozeß gewährleistet ist und andererseits auch eine wirksame Ausübung der Schlichter-Rolle sichergestellt werden kann.

Die Qualifikationsanforderungen an einen Schlichter sind vor allem:

- reiche Erfahrungen in Schlichtungs-Angelegenheiten,

- hoher sozialer Status (Prestige, Elitenzugehörigkeit oder hoher Status in Verwandtschaft),

- Repräsentant von Normen und Werten in der Gemeinschaft (z. B. Monkalun bei den Ifugao), reichhaltige Kenntnisse über das Gemeinschaftsleben (Verwandtschaftsbeziehungen, Reputationen),

neutrale, aber nicht uninteressierte Haltung zum jeweiligen Konflikt.

b) Die Entscheidung für ein Schlichtungs-Verfahren

Ob zur Bearbeitung eines Konflikts gerade eine Schlichtung gewählt wird, hängt - wie bereits aus dem paradigmatischen Ansatz bekannt - von einer ganzen Reihe von Umständen ab; Merry $^{74}$ betont vor allem:

- den Zukunftsbezug der Sozialbeziehung (bei den Nuer hat Mord Rache zur Folge, es sei denn, der Mörder ist mit den Opfern eng verwandt oder nahe zusammenlebend, dann wird eine Schlichtung versucht)

- die Einflußnahme der Verwandtschaft, die auf eine friedliche Beilegung des Konflikts drängt (,phenomenon of community" ${ }^{75}$ ); vor allem, wenn die einzige Alternative zur Schlichtung die Rache darstellt und damit der Konflikt andere Gemeindeangehörige in Mitleidenschaft zieht;

- die Furcht vor sozialer Isolation bei Inanspruchnahme der Alternative ,Gericht' und

- die Statusrelation zwischen den Streitparteien ${ }^{76}$.

c) Der mit Schlichtung verbundene Zwangscharakter

Schlichtung ist in den untersuchten small-scale-Gesellschaften stets eingebettet in einen Sanktions- und Kontrollkontext, der die Einhaltung bzw. faktische Realisierung des Schlichtungs-Ergebnisses sicherstellt. Der Konsensfindungsprozeß im Schlichtungsverfahren braucht die für das Ergebnis erforderliche Stabilität also nicht aus sich selbst zu 
entwickeln, sondern kann sich auf soziale Ressourcen stützen. Die Kontrollen können erfolgen:

- durch den Schlichter, der entweder selbst über Waffengewalt verfügt ( $z$. B. Monkalun) oder die politische oder ökonomische Macht seines Clans aktivieren kann, oder indem er mit Gericht droht (,... in the shadowing of the law");

- durch die Gemeinschaft, sei es informell durch ,gossip and scandal", durch die Auslegung bestimmter kultureller Standards bei starrem Verhandlungsverhalten (Gesichtsverlust in China), durch den Entzug materieller Unterstützung;

- durch das kulturelle Wertsystem, das die Streitenden internalisiert haben (Furcht vor Verzauberung bei Hartnäckigkeit (Zinacatan)) oder letztlich

- durch fehlende Alternativen zum Schlichtungs-Verfahren (abgesehen von Rache oder Isolation, s. o.).

d) Die Qualität des Resultats

Nach den bei Merry berichteten Befunden gilt in small-scale-Gesellschaften, daß das in Schlichtungsverfahren erzielte Resultat die bestehenden Sozialbeziehungen der Streitenden widerspiegelt; bei der Ergebnissuche orientiert man sich nicht etwa an dem Grundsatz "gleiches Recht für alle", sondern stellt die jeweilige konkrete soziale Identität der Beteiligten und ihrer Familien in Rechnung (so gilt z. B. für die Waigali, daß das Schlichtungs-Ergebnis den sozialen Status des Geschädigten demonstrieren muß); das bedeutet, daß bei ungleicher Sozialbeziehung auch das Ergebnis ungleich ausfällt und eines ist, das den Schwächeren benachteiligt (z. B. Ifugao, Nuer, Waigali). Allerdings betont Merry ${ }^{77}$, daß die Berücksichtigung der konkreten sozialen Identitäten der Parteien umso geringer ausfällt, desto mächtiger der Schlichter ist.

Ein weiteres wichtiges Merkmal des Ergebnis-Charakters liegt in der Unmittelbarkeit des Ergebnis-Vollzuges (dies natürlich v. a. in Gemeinschaften ohne Schrift) und in der Konkretheit seiner Formulierung; ,vague promises of improved behavior in the future are not sufficient" ${ }^{78}$.

\section{e) Der Schlichtungs-Prozeß}

Merry ${ }^{79}$ betont folgende 4 Prozeß-Merkmale:

- Kontiguität: In der Regel findet das Schlichtungs-Verfahren unmittelbar nach Entstehung des Konflikts statt.

- Zeitaufwendigkeit: Für die Bearbeitung des Konflikts nimmt man sich Zeit; bei den Waigali findet sich allerdings ein interessanter selbstregulierender Mechanismus: der Schlichter, der du-wrai, wird während des Verfahrens von den Parteien bewirtet und zwar mit ausgesprochen

77 Merry (N. 73), 33.

78 Ebd., 29.

79 Ebd., 29--30. 
erlesenen und teuren Köstlichkeiten; mit zunehmender Verfahrensdauer steigen entsprechend die Kosten; kommt es nicht zu einer Einigung, zieht der du-wrai einen Kollegen aus dem Nachbardorf hinzu, wodurch sich die Parteien nicht nur dessen Spott einhandeln, sondern auch die Kosten für eine doppelte Bewirtung.

- Publizität: Die Schlichtungs-Verfahren sind in den untersuchten Gemeinschaften in der Regel öffentlich.

- Ritualisierung: Diese betrifft vor allem die Phase der Ergebnis-Sicherung, also nach erfolgter Einigung, die ihren Abschluß meist in einer gemeinsamen Zeremonie findet (Kaffeetrinken im Libanon, Fest in China).

Insgesamt zeigen die Befunde bei Merry, daß das Funktionieren von Schlichtung wesentlich gebunden ist an:

- die Stabilität der umgebenden sozialen Struktur (soziale Identitäten), die Schlichtung erst zuläßt und die durch sie reproduziert wird;

- den Konsens über ein geteiltes Wert-System, der das Spektrum des Normalen und Ublichen beschränkt;

- ein hohes Ausmaß an Kontroll- und Einflußmöglichkeiten auf den Einzelnen, das Erfüllung von Vereinbarungen sicherstellt,

- und ein restriktives Spektrum alternativer Möglichkeiten der Konfliktbearbeitung, vor allem dann, wenn sie mit Gefahr für Leib und Leben verbunden sind.

Die spezifische Leistungsfähigkeit von Schlichtung, so läßt sich insgesamt als empirisch begründete Hypothese folgern, beruht auf den Besonderheiten seiner interaktiven Ausgestaltung nur unter der Randbedingung, daß diesen ein bestimmtes ,,soziales equipment" beigegeben ist. Dieses soziale equipment bindet das kommunikative Geschehen in die sozialen Strukturen der jeweiligen Gesellschaft ein, liefert die Voraussetzung für den Verfahrensvollzug und wird seinerseits durch die Schlichtungs-Prozesse reproduziert.

Es versteht sich übrigens, daß unter Annahme einer solchen Hypothese Überlegungen zur Übertragung von Schlichtungs-Verfahren aus small-scale-Gesellschaften mit begründeter Skepsis begegnet wird.

Eine Studie, die das Zusammenspiel unterschiedlicher Handlungsbedingungen auf die Wahl von Schlichtung als Verfahren der Konflikt-Bearbeitung deutlich macht, liegt für das ländliche China vor ${ }^{80}$. Cohen stellt fest, daß es trotz umfassender Prozeßverfahrensvorschriften für die Menschen im ländlichen China eine kulturelle Präferenz für Schlichtung gibt, und führt die Bedingungen an, die diese Präferenz erzeugen:

eine kulturell-moralische Orientierung am Konfuzianismus, derzufolge die Herstellung von Harmonie und das Funktionieren sozialer Ordnung hohe Priorität bestitzt. Die ideale Regelung von Konflikt erfolgt durch Selbstkritik: Man kritisiert das eigene Verhalten und verpflichtet den Anderen damit, sein eigenes Handeln ebenfalls zu kritisieren; dadurch löst sich der Konflikt auf;

die staatlich-administrativen Verhältnisse, insbesondere die Oberbelastung der VerwaltungsBürokratien; 
- die sozialen Verbände, in denen sich die Parteien befinden (Familie, Dorfgemeinschaft, Clan oder Gilde), die von der Konfliktgeschichte mitbetroffen sind und nach Maßgabe eigener Interessen intervenieren (Vermeiden von Eskalationen);

- die individuelle Kosten-Nutzen-Perspektive, die Für und Wider der Schlichtung kalkuliert. Für das nur infrage kommende Gerichtsverfahren gilt: die Gerichtskosten sind hoch, die Verfahrensdauer ist lang, die Richter gelten als korrupt und der Ausgang ist ungewiß.

Diese unterschiedlichen Bedingungen stützen sich in ihrer Wirksamkeit gegenseitig, v. a. dient die moralische Orientierung und die Überbelastung dem Staatsapparat zur Legitimierung des Nicht-Agierens und fördert damit die interne Kohärenz der sozialen Verbände ${ }^{81}$.

Die Studien von Merry und Cohen zeigen, daß die Funktionalität von Schlichtung davon abhängt, daß ein entsprechend verfahrens-unterstützender kultureller Kontext zur Verfügung gestellt wird, auf dessen „Boden“ das einzelne Schlichtungs-Geschehen eine faktische interaktive Wirksamkeit entfalten kann.

\section{Abscbließende Einscbätzung}

Zusammenfassungen von Ergebnissen tendieren dazu, die Reichhaltigkeit derselben wieder auszudünnen und in Algemeinplätzen zu münden. Statt einer Zusammenfassung soll abschließend die Frage andiskutiert werden, welche Implikationen diese Ergebnisse für eine gesprächsanalytische Bestimmung von Schlichtung als ein komplexes Interaktionsmuster haben. In dieser Hinsicht sind die Befunde ermutigend und zugleich herausfordernd: Ermutigend, weil es offenbar einen „, harten kommunikativen Kern" von Schlichtung gibt, von dem aus die Gesprächsanalyse von Schlichtung valide fortschreiten kann (insbesondere Gulliver); herausfordernd, weil die Befunde eine gesprächsanalytische Bestimmung von Schlichtung in ihre Schranken weist: zeigt sich doch, daß das Schlichtungsgeschehen wesentlich von situationsübergreifenden Bezügen gesteuert wird (Konflikt-Syntagma, Verfahrensparadigma), und daß das SchlichtungsVerfahren in seinem Funktionieren von der Aktivierung übergeordneter sozial-struktureller Bedingungen abhängt. Das Musterhafte von Schlichtung, so läßt sich als begründete Hypothese formulieren, liegt nur zum Teil im interaktiven Verlauf selbst, zum anderen Teil in Parametern des kulturellen Kontext, in den Schlichtungs-Interaktion eingebunden ist.

81 Kennzeichnend für die staatliche Haltung der Inanspruchnahme von Gerichten gegenüber ist folgende Äußerung des chinesischen Kaisers K'ang-shi (1662-1722):

,.... lawsuits would tend to increase to a frighful amount, if people were not afraid of the tribunals, and if they felt confident of always finding in them ready and perfect justice. As man is apt to delude himself concerning his hown interests, contests would then be interminable, and the half of the Empire would not suffice to settle the lawsuits of the other half. I desire, therefore, that those who have recourse to the tribunals should be treated without any pity, and in such a manner that they shall be disguisted with law, and tremble to appear before a magistrate." (zitiert nach Cohen (N. 16), 66). 